\title{
Satisfação no trabalho de técnicos de enfermagem do atendimento pré-hospitalar: um estudo observacional analítico
}

\author{
Job satisfaction of nursing technicians in pre-hospital care: an analytical observational study
}

Satisfacción laboral de los técnicos de enfermería en la atención prehospitalaria: un estudio analítico observacional

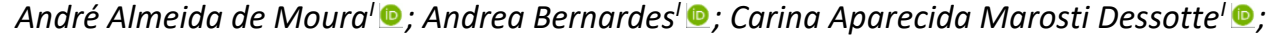 \\ Alexandre Pazetto Balsanellil ${ }^{\circledR}$; Ariane Cristina Barboza Zanettil @
}

'Universidade de São Paulo, Ribeirão Preto, SP, Brasil; "Universidade Federal de São Paulo, São Paulo, SP, Brasil

\begin{abstract}
RESUMO
Objetivo: avaliar a satisfação no trabalho dos técnicos de enfermagem atuantes no Serviço de Atendimento Móvel de Urgência e sua relação com variáveis relacionadas aos aspectos profissionais e demográficos. Método: estudo observacional analítico, com 155 técnicos de enfermagem do Serviço de Atendimento Móvel de Urgência. Empregaram-se o Job Satisfaction Survey e testes estatísticos não paramétricos para analisar a correlação e a associação entre as dimensões do instrumento com as variáveis sociodemográficas e profissionais, com nível de significância de $p<0,05$. Resultados: os profissionais estão nem insatisfeitos nem satisfeitos em relação ao seu trabalho. Identificaram-se correlações fracas entre alguns domínios do instrumento com as variáveis idade e tempo na unidade. Houve associações entre a satisfação no trabalho com as variáveis: sexo, graduação, escala e tipo de vínculo. Conclusão: evidenciou-se que a satisfação no trabalho dos técnicos de enfermagem atuantes no atendimento pré-hospitalar está associada às variáveis sexo, graduação, escala de serviço e tipo de vínculo. Descritores: Enfermagem; Emergências; Administração de Recursos Humanos; Serviços Médicos de Emergência; Satisfação no Trabalho.
\end{abstract}

\begin{abstract}
Objective: to assess job satisfaction among nursing technicians working in a Mobile Emergency Care Service and its relationship with professional and demographic variables. Method: in this analytical observational study of 155 nursing technicians from the Mobile Emergency Care Service, the Job Satisfaction Survey and non-parametric statistical tests were used to examine correlations and associations between the dimensions of the instrument and sociodemographic and professional variables, to a $p<0.05$ level of significance. Results: the technicians were neither dissatisfied nor satisfied with their work. Weak correlations were identified between some domains of the instrument, age and time in the unit. Job satisfaction was associated with sex, graduation, scale of service and type of employment relationship. Conclusion: job satisfaction among nursing technicians working in pre-hospital care was found to be associated with sex, graduation, scale of service and type of employment relationship.
\end{abstract}

Descriptors: Nursing; Emergencies; Personnel Management; Emergency Medical Services; Job Satisfaction.

\section{RESUMEN}

Objetivo: evaluar la satisfacción no trabajo de los técnicos de enfermería que trabajan en el Servicio de Atención Móvil de Urgencias y su relación con variables relacionadas con los aspectos profesionales y demográficos. Método: estudio analítico observacional junto a 155 técnicos de enfermería del Servicio de Atención Móvil de Urgencias. Se utilizaron la Encuesta de Satisfacción Laboral y pruebas estadísticas no paramétricas para analizar la correlación y la asociación entre las dimensiones del instrumento y las variables sociodemográficas y profesionales, con el nivel de significancia de $p<0.05$. Resultados: los profesionales no están insatisfechos ni satisfechos con respecto a su trabajo. Se identificaron correlaciones débiles entre algunos dominios del instrumento con las variables edad y tiempo en la unidad. Hubo asociaciones entre la satisfacción laboral con las variables: sexo, graduación, escala y tipo de vínculo laboral. Conclusión: se evidenció que la satisfacción laboral de los técnicos de enfermería que laboran en la atención prehospitalaria se asocia con las variables sexo, grado, escala de servicio y tipo de vínculo.

Descriptores: Enfermería; Urgencias Medicas; Administración de Personal; Servicios Médicos de Urgencia; Satisfacción em el Trabajo.

\section{INTRODUÇÃO}

A satisfação no trabalho constitui um dos componentes mais estudados acerca do comportamento organizacional, sendo considerado um importante preditor de bem-estar no ambiente de trabalho ${ }^{1}$. Na enfermagem, tal variável está relacionada a aspectos como desempenho, qualidade de vida e/ou condição de saúde dos profissionais ${ }^{1,2}$, bem como impacta na qualidade do atendimento e na satisfação do paciente ${ }^{2,3}$.

Dessa forma, a implementação de programas e de políticas de saúde que visam à melhoria e ao desenvolvimento da satisfação no trabalho contribuem para a saúde desses profissionais e consequentemente dos pacientes que recebem os cuidados de enfermagem ${ }^{4}$ 
Cabe apontar que não existe consenso no que concerne à definição de satisfação no trabalho, tendo em vista que existem distintas teorias que a abordam, sob diversas perspectivas ${ }^{5}$. O conceito mundialmente mais difundido e utilizado no presente estudo, a define como um estado emocional positivo ou agradável resultante da avaliação de suas experiências de trabalho ou de emprego. De acordo com o referido conceito, os profissionais formam suas atitudes em relação aos seus empregos, considerando tanto seus sentimentos quanto suas crenças ${ }^{6}$.

Diante desse referencial, nota-se que a satisfação no trabalho é uma questão complexa, multifacetada e não linear ${ }^{3}$. Assim, a investigação acerca da satisfação no trabalho da equipe de enfermagem pode favorecer a identificação de problemas nos serviços de saúde, o planejamento de possíveis soluções e, por conseguinte, a produção de avanços no ambiente de trabalho e na qualidade dos serviços.

No que tange aos profissionais de enfermagem, torna-se válido notar a colaboração dos técnicos de enfermagem, cuja formação ocorre por meio de cursos pertencentes à educação profissional técnica de nível médio. Tal habilitação profissional técnica capacita essa categoria profissional de realizar procedimentos e cuidados em enfermagem de baixa e média complexidades. Ademais, esses profissionais compreendem uma parcela significativa da força de trabalho em saúde e da enfermagem no Brasil. Pesquisa recente apresentou que $77 \%$ dos profissionais da área no país correspondem a técnicos ou a auxiliares de enfermagem ${ }^{7,8}$.

Dado o montante expressivo dessa classe profissional e considerando-se os inúmeros cenários de atuação, no Serviço de Atendimento Móvel de Urgência (SAMU) os técnicos de enfermagem desempenham uma função fundamental. O SAMU compreende o componente móvel de urgência normativamente instituído no Brasil e consiste no serviço de socorro pré-hospitalar, em que o usuário solicita atendimento por intermédio do acesso telefônico gratuito, contemplado por um componente regulador (Central Médica de Regulação) e por um componente assistencial (equipe das ambulâncias). Os técnicos que compõem a equipe desse serviço prestam os cuidados nas unidades de suporte básico de vida, atividades consideradas complexas - e em determinados momentos estressantes - a ponto de interferirem diretamente na sua satisfação no trabalho ${ }^{9,10}$.

Estudos apontam que ao considerar a complexidade do cenário de atuação dos profissionais de enfermagem, neste caso especificamente o atendimento móvel de urgência, inúmeros fatores têm influência na satisfação no trabalho ${ }^{9,11,12}$, exemplo a liderança exercida pelo enfermeiro ${ }^{9,11}$. Dessa forma, novas investigações acerca da satisfação no trabalho dos técnicos de enfermagem, como o presente trabalho, são relevantes, de maneira a mitigar complicações como: estresse, desgaste físico e emocional da equipe ${ }^{9,11-14}$. No entanto, cabe apontar ainda que é incipiente o número de trabalhos que analisa a satisfação no trabalho dos profissionais nesse contexto, especialmente dos técnicos de enfermagem ${ }^{9,11-14}$

Desse modo, é de grande valor identificar a satisfação dos técnicos de enfermagem dentro do contexto de trabalho do referido serviço, razão pela qual o presente trabalho buscou avaliar a satisfação no trabalho dos técnicos de enfermagem atuantes no Serviço de Atendimento Móvel de Urgência, bem como investigar sua possível relação com aspectos profissionais e demográficos.

\section{MÉTODO}

Trata-se de estudo observacional analítico, do tipo transversal realizado com técnicos de enfermagem em 11 das 12 unidades das Centrais de Regulação do Serviço de Atendimento Móvel de Urgência (SAMU) do Estado de Goiás, Brasil. Trata-se de uma amostra por conveniência composta por 210 técnicos de enfermagem.

Adotaram-se como critérios de inclusão: técnicos de enfermagem em exercício nas unidades há pelo menos seis meses, considerando-se esse período mínimo para ambientação do profissional. Foram excluídos os profissionais que estivessem em gozo de férias, de licença-prêmio ou em afastamento por motivos de saúde no período da coleta de dados.

Para obtenção dos dados referentes à satisfação no trabalho foi adotado o instrumento Job Satisfaction Survey $(\mathrm{JSS})^{15}$, na sua versão adaptada para o Brasil ${ }^{5}$. Tal instrumento é composto por 36 itens, distribuídos em nove domínios: Pagamento (itens 1, 10, 19 e 28); Promoção (itens 2, 11, 20 e 33); Supervisão (itens 3, 12, 21 e 30); Benefícios (itens 4, 13, 22 e 29); Recompensas (itens 5, 14, 23 e 32); Procedimentos Operacionais (itens 6, 15, 24 e 31); Colaboradores (itens 7, 16, 25 e 34); Natureza do serviço (itens 8, 17, 27 e 35) e; Comunicação (itens 9, 18, 26, 36). Cada domínio possui quatro itens avaliados por uma escala do tipo Likert de seis pontos que pode variar segundo a gradação: discordo muito (1); discordo moderadamente (2); discordo pouco (3); concordo pouco (4); concordo moderadamente (5) e; concordo muito (6). A avaliação é feita por meio da soma de cada item, sendo possível obter, portanto, um escore para cada domínio que varia entre 4 e 24 pontos. Dessa forma, o escore total da escala pode variar de 36 a 216 pontos, de maneira que valores entre: 36 e 108 indicam insatisfação; 144 a 216 denotam satisfação e; 109 e 143 não indicam satisfação nem insatisfação ${ }^{5,16}$. 
Foram também coletados dados de caracterização demográfica e aspectos funcionais, a saber: idade (resultado, em anos, da subtração entre a data da entrevista e a data de nascimento); tempo de formação (resultado, em anos, da diferença entre a data da entrevista e a data de conclusão do curso); tempo na unidade (resultado, em anos, da subtração entre a data da entrevista e a data de admissão na unidade do SAMU); sexo (masculino e feminino); tipo de vínculo (celetista, estatutário, contrato e outros); tipo de escala (12 horas de trabalho e 60 de descanso; 24 horas de trabalho com 72 horas de descanso e; 12 horas de trabalho e 36 horas de descanso); possuir curso complementar (sim e não) e; ter concluído graduação (sim e não).

A coleta dos dados transcorreu entre os meses de abril e agosto de 2017, por um dos pesquisadores, responsável por entregar os instrumentos durante as trocas de plantões e que foram preenchidos pelos próprios técnicos de enfermagem, nas próprias unidades do SAMU. A posteriori os instrumentos foram recolhidos pelo mesmo pesquisador em datas e horários previamente agendados. Foi assegurado que os participantes preenchessem por completo os itens presentes nos questionários. Os dados coletados foram duplamente digitados em planilhas distintas e, posteriormente, realizada a validação da base de dados. Em seguida, os dados foram transportados para Programa IBM-SPSS, versão 22.0 para Windows, responsável por realizar a análise dos dados (SPSS, Inc., Chicago, IL, USA).

Para a avaliação descritiva das variáveis, foram realizadas análises de frequência simples para variáveis nominais ou categóricas, e análise de tendência central (média e mediana) e de dispersão (desvio-padrão) para as variáveis contínuas. Para avaliar a correlação do escore total e dos escores dos nove domínios do JSS com a idade, tempo de formação e tempo na unidade, foi utilizado o Teste de Correlação de Spearman. Ainda, para as correlações entre as variáveis presentes no estudo, adotou-se o poder da correlação variando de muito alto $(0,9$ a 1,0 ou $-0,9)$ a fraco $(0,0$ a $0,3$ ou 0,0 a $-0,3)^{17}$.

Adicionalmente, para investigar as associações do escore total e dos escores dos nove domínios do JSS com o sexo, formação complementar e ter graduação, empregou-se o Teste de Mann-Whitney. Por fim, para avaliar os escores do instrumento com a escala de trabalho e tipo de vínculo, utilizou o teste de Kruskal-Wallis. O nível de significância foi de $5 \%$.

A presente pesquisa foi apreciada e aprovada pelo Comitê de Ética em Pesquisa da Escola de Enfermagem de Ribeirão Preto/USP, sob o número 046/2017 e todos os participantes assinaram o Termo de Consentimento Livre e Esclarecido.

\section{RESULTADOS}

De uma população de 210 profissionais, 155 consentiram participaram do presente estudo, representando um percentual de $73,80 \%$. Os demais profissionais, ou seja, os $36,20 \%$ dos técnicos de enfermagem das unidades pesquisadas, correspondiam aos participantes que não atendiam aos critérios da pesquisa $(n=48)$ ou não aceitaram participar da pesquisa $(n=7)$. A média de idade dos participantes da pesquisa foi de 39,26 anos, com o desvio-padrão $(D P)=8,14$; a média em relação ao tempo de formação foi de 12,65 anos $(D P=6,17)$; e o tempo de serviço médio na unidade era de 6,16 anos (DP=3,69).

A maior parte desses técnicos $(n=95 ; 61,29 \%)$ era do sexo feminino, possuía como vínculo empregatício o regime estatutário ( $n=94 ; 60,65 \%$ ) e executava escala de trabalho de 12 horas com 60 horas de intervalo de descanso ( $n=114$; $73,54 \%)$. Ademais, cabe ressaltar que $87(56,13 \%)$ técnicos possuíam curso complementar (como instrumentação cirúrgica, técnico de enfermagem de segurança no trabalho, entre outros) e 95 desses profissionais (61,29\%) eram graduados nas mais diversas áreas de formação superior.

Tendo em vista o questionário JSS aplicado e com base na classificação do nível de satisfação no trabalho, verificouse que esses profissionais se sentem nem insatisfeitos e nem satisfeitos no trabalho, haja vista que a média do escore total da escala foi 124,24 , com DP de 21,5 e mediana de 124 .

De acordo com o mesmo instrumento, as duas dimensões que apresentaram maiores porcentagens de satisfação no trabalho na ordem decrescente foram Natureza do serviço 150 (96,77\%) e Supervisão 128 (82,58\%). Em relação à insatisfação, as dimensões presentes no instrumento que apresentaram maiores percentuais de insatisfação foram Pagamento 140 (90,32\%) e Benefícios 132 (85,16\%). A Tabela 1 apresenta as correlações (Spearman e o $p$-valor) entre os domínios da satisfação no trabalho com as variáveis quantitativas: idade, tempo de formação e tempo na instituição.

Observa-se pelos resultados presentes nas Tabelas 1 que houve correlação negativa fraca entre o domínio "Recompensa" do instrumento de satisfação com a variável tempo na unidade. Também foi evidenciada correlação igualmente fraca entre o domínio "Colaboradores" do JSS com a variável idade. As Tabelas 2 e 3 apresentam as associações entre as dimensões da satisfação no trabalho com as demais variáveis em análise na pesquisa. 
TABELA 1: Correlação de Spearman entre as dimensões da satisfação no trabalho e as variáveis idade, tempo de formação e tempo na unidade dos técnicos de enfermagem do SAMU ( $n=155)$. Goiás, GO, Brasil, 2017

\begin{tabular}{lcccccc}
\hline \multirow{2}{*}{ Dimensão } & \multicolumn{2}{c}{ Idade } & \multicolumn{2}{c}{ Tempo de formação } & \multicolumn{2}{c}{ Tempo na unidade } \\
& Spearman & $\boldsymbol{p}$-valor & Spearman & $\boldsymbol{p}$-valor & Spearman & $\boldsymbol{p}$-valor \\
\hline Pagamento & 0,058 & 0,474 & $-0,011$ & 0,89 & $-0,054$ & 0,501 \\
Promoção & 0,053 & 0,51 & $-0,054$ & 0,505 & $-0,157$ & 0,052 \\
Supervisão & 0,088 & 0,277 & $-0,072$ & 0,376 & 0,069 & 0,393 \\
Benefícios & 0,049 & 0,542 & $-0,126$ & 0,12 & $-0,063$ & 0,436 \\
Recompensa & $-0,107$ & 0,184 & $-0,142$ & 0,078 & $-0,233$ & 0,003 \\
Protocolos Operacionais & 0,084 & 0,299 & $-0,031$ & 0,703 & $-0,081$ & 0,315 \\
Colaboradores & 0,187 & 0,02 & 0,026 & 0,747 & 0,007 & 0,934 \\
Natureza do serviço & 0,034 & 0,676 & 0,053 & 0,509 & $-0,011$ & 0,889 \\
Comunicação & 0,062 & 0,441 & $-0,094$ & 0,247 & $-0,122$ & 0,13 \\
\hline Escore total da escala & 0,087 & 0,284 & $-0,1$ & 0,214 & $-0,137$ & 0,09 \\
\hline
\end{tabular}

TABELA 2: Associação entre as variáveis sexo, ter curso complementar e ter graduação com os domínios da satisfação no trabalho ( $n=155)$, Goiás, Brasil, 2017

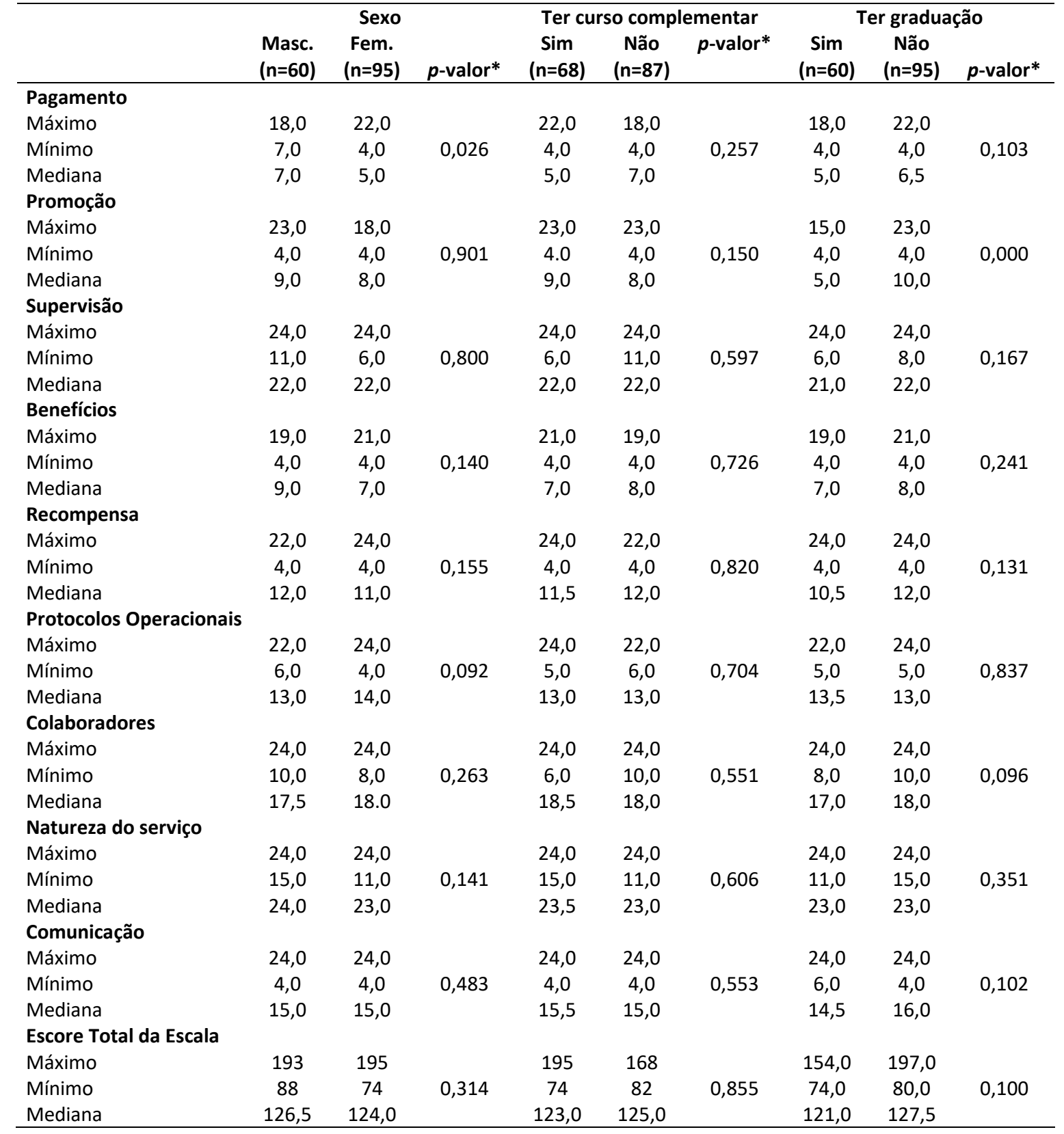

* $p$-valor referente ao Teste $\mathrm{U}$ de Mann-Whitney. 
No que tange à categoria sexo, houve diferença estatisticamente significativa apenas na dimensão Pagamento, posto que o sexo masculino apresentou maior satisfação, em comparação com o sexo feminino. Não foi identificado associação entre a satisfação no trabalho (dimensões e escore total da escala) com a variável ter curso complementar. Já a variável possuir graduação apresentou associação estatisticamente significativa com a dimensão Promoção do instrumento JSS.

TABELA 3: Associação entre as variáveis escala de serviço, tipo de vínculo com os domínios da satisfação no trabalho (n=155), Goiás, Brasil, 2017

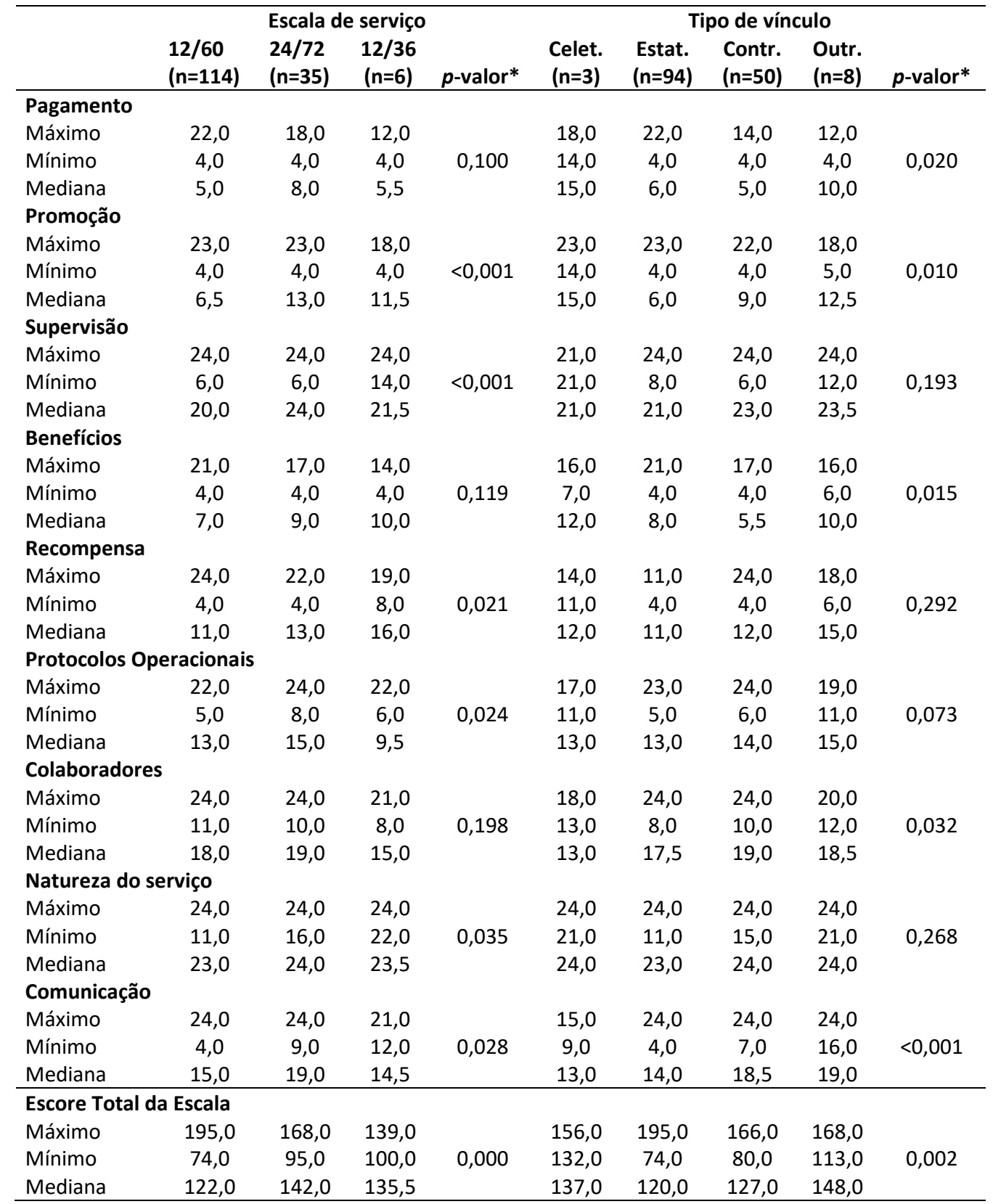

* $p$-valor referente ao Teste Kruskal-Wallis.

A Tabela 3 demonstra que houve associação entre escalas de serviço com praticamente todas as dimensões da satisfação do trabalho do JSS, especialmente quando analisadas a escala de 12 horas de trabalho com 60 de descanso e a escala de 24 horas de trabalho com 72 horas de descanso, como descritos nos $p$-valores: Escore Total da Escala $(<0,001)$; Promoção $(<0,001)$; Supervisão $(<0,001)$; Recompensa $(0,019)$; Protocolos Operacionais $(0,044)$; Natureza do serviço $(0,039)$ e; Comunicação $(0,022)$. 
Esses valores indicam que os profissionais que trabalham em uma escala de 24 horas com 72 horas de descanso estão mais satisfeitos que os profissionais que desenvolvem uma escala de 12/60 nos domínios: Promoção; Supervisão; Recompensa; Natureza do serviço e Comunicação, além do Escore total da Escala. Em relação aos Protocolos Operacionais, os técnicos de enfermagem que executam a escala de 12 horas trabalhadas por 60 de descanso sentemse mais satisfeitos quando comparados àqueles que realizam a escala 24/72.

Quanto ao tipo de vínculo empregatício, também se evidenciou uma diferença estatisticamente significativa entre as dimensões do JSS com as quatro formas de vínculos. Nessa análise, em específico, cabe ressaltar as associações evidenciadas pelo vínculo empregatício estatuário (concursado) com os demais, principalmente quando comparado ao vínculo "outros" (vínculo comissionado). Encontrou-se um valor estatisticamente significativo nos domínios Promoção $(p-0,034)$, Comunicação $(p-0,014)$ e Escore Total da Escala $(p-0,024)$, denotando assim que os profissionais concursados estavam mais insatisfeitos do que os profissionais comissionados em relação aos mesmos domínios mencionados.

\section{DISCUSSÃO}

É possível verificar pelos resultados gerais dos domínios que compõem a satisfação no trabalho que a categoria profissional de enfermagem analisada se apresenta nem insatisfeita nem satisfeita em relação ao trabalho. Estudos internacionais que utilizaram o mesmo questionário para analisar a satisfação no trabalho de enfermeiros e/ou de equipes de enfermagem apontam faixas semelhantes de satisfação e ambivalência desses profissionais em relação à satisfação no trabalho ${ }^{18-20}$

Destarte, como forma de favorecer a satisfação no trabalho, estudos recentes identificaram que modelos de liderança, a exemplo das lideranças autêntica, ressonante e coaching, apresentam correlação positiva na satisfação da equipe de enfermagem de um modo geral ${ }^{21-23}$, bem como na equipe de técnicos de enfermagem do SAMU ${ }^{24}$. Portanto, tal competência deve ser potencialmente desenvolvida pelos supervisores e pelos coordenadores de enfermagem, com vistas a impactar na satisfação no trabalho das equipes de enfermagem e de saúde.

Analisando ainda os dados descritivos, observou-se que na percepção dos técnicos de enfermagem do SAMU, Natureza do serviço e Supervisão foram os domínios que apresentaram maior satisfação no ambiente de trabalho, corroborando assim estudos que utilizaram o mesmo instrumento ${ }^{19,25}$. Perante esses dados e considerando o contexto da urgência e da emergência, cabe apontar pesquisa de cunho qualitativo desenvolvida com técnicos de enfermagem que ressaltou que o prazer que esses profissionais sentem no seu trabalho está atrelado às atividades que executam na sua rotina diária ${ }^{13}$.

Nessa mesma lógica, destaca-se também estudo desenvolvido no SAMU da cidade de Natal, Rio Grande do Norte, Brasil, com profissionais de enfermagem, dentre os quais $90,2 \%$ responderam estar satisfeitos com os tipos de atividades que desenvolvem no seu cotidiano de trabalho ${ }^{9}$. Da mesma forma, estudo desenvolvido no serviço equivalente ao SAMU no Chile desvelou que profissionais de enfermagem entrevistados destacaram a natureza do serviço como principal componente para maior satisfação ${ }^{26}$. Ainda, considerando-se o contexto do serviço móvel de urgência, cabe citar que, além dos fatores evidenciados nesta pesquisa, foram identificados como fatores que promovem satisfação na equipe de enfermagem do SAMU: o dinamismo, a resolubilidade do serviço e a visibilidade na rede de atenção às urgências ${ }^{12}$.

No tocante à Supervisão, é notório ressaltar que compete ao enfermeiro a função de supervisionar a equipe de enfermagem, uma vez que esse profissional é responsável pela gestão do serviço de enfermagem junto ao órgão fiscalizador do exercício profissional, articulando para tanto dimensões profissional, institucional e social, do país e do mundo ${ }^{27}$. Oportuno mencionar nesse sentido que, diante dos resultados do presente estudo, os profissionais técnicos se sentem satisfeitos com a supervisão realizada por seus coordenadores.

A insatisfação pode estar atrelada a uma ou mais dimensões bem como variar de acordo com os contextos social e temporal. No presente trabalho, a dimensão Pagamento foi responsável pelo maior valor de frequência de insatisfação 140 (90,32\%), fato que corrobora pesquisa realizada com enfermeiros italianos que apresentou a mesma associação, embora com valores ainda menores de satisfação no instrumento JSS ${ }^{25}$.

Os dados da Tabela 1 demonstraram que as variáveis quantitativas (idade, tempo de formação e tempo na unidade) não apresentaram correlação forte com a satisfação no trabalho. Em contrapartida, duas revisões integrativas sobre os fatores que interferem na satisfação da enfermagem indicaram relação entre essas variáveis. Em ambas relacionaram um número artigos que demonstram que o tempo de formação exerce correlação positiva para a satisfação no trabalho, ou seja, quanto maior o tempo de formação e o tempo no cargo, maior a satisfação no trabalho ${ }^{28,29}$. Por sua vez, no que tange à análise da idade, a revisão acerca dos profissionais da enfermagem na atenção primária descreveu que indivíduos mais jovens sentem menor satisfação no seu trabalho comparados aos mais velhos ${ }^{28}$, o que pode estar associado à maturidade do profissional em reconhecer os fatores intervenientes na satisfação do trabalho. 
Ao considerar os dados da Tabela 2, foi identificado associação entre a variável sexo e a dimensão Pagamento, uma vez que profissionais do sexo masculino mostraram-se mais satisfeitos quando comparados ao sexo feminino, tal qual artigo decorrente de pesquisa em hospitais no sul do Brasil ${ }^{30}$

Ainda sobre os dados da Tabela 2, não foi observada a associação entre curso complementar dos técnicos com as dimensões da satisfação no trabalho. Não ter graduação para os técnicos estava associada à satisfação desses profissionais quanto ao domínio promoção. Contrapondo o quanto exposto no presente trabalho, estudo chinês apontou que o nível de escolaridade é uma variável que pode ter associação com a satisfação no trabalho, posto que os dados sinalizam que quanto maior o nível de escolaridade e de formação (como os cursos complementares), maior a satisfação no trabalho dos profissionais de enfermagem².

Na Tabela 3, quando analisadas as escalas de trabalho e as dimensões do JSS, houve diferença estatisticamente significativa no que tange a escala de $12 / 60$ e a escala 24/72, diferentemente de revisão sistemática que não identificou que a escala de trabalho tem correlação com a satisfação no trabalho de profissionais de enfermagem ${ }^{29}$. De maneira contrária aos achados do presente estudo, pesquisa brasileira apontou que os trabalhadores concursados, ou seja, aqueles que apresentam estabilidade no emprego, dispõem de plano de cargos e salários, fato este que pode influenciar positivamente na satisfação dos trabalhadores ${ }^{30}$.

Observa-se, portanto, que a satisfação no trabalho tem um papel fundamental para a realização de boas práticas de enfermagem ao possibilitar um ambiente favorável para elas. Sendo assim, é essencial avaliar os fatores que podem (des)favorecer a satisfação no trabalho dos técnicos de enfermagem. Finalmente, importante destacar que o presente estudo apresenta como limitação a escolha das unidades do SAMU alocadas nas centrais de regulação do Estado de Goiás, não sendo assim estendidos os resultados para as bases descentralizadas, razão pela qual se justifica a relevância da realização desta pesquisa e de outras de maneira a robustecer o conhecimento gerado acerca da temática na área.

\section{CONCLUSÃO}

Os técnicos de enfermagem das unidades do SAMU analisadas demonstraram que estão nem insatisfeitos nem satisfeitos em relação ao seu trabalho. Além disso, os aspectos que apresentaram maior satisfação para os profissionais foram Natureza do serviço e Supervisão, ao passo que a dimensão Pagamento foi identificada como maior geradora de insatisfação.

Ao investigar sua possível relação com aspectos profissionais e demográficos, evidenciou-se correlação negativa fraca entre o domínio Recompensa com a variável tempo na unidade, assim como entre o domínio Colaboradores com a variável idade. Em contrapartida, constatou-se associação positiva entre escalas de serviço com praticamente todas as dimensões da satisfação do trabalho do JSS, especialmente quando analisadas a escala de 12/60 e a escala 24/72. Os quatro tipos de vínculo empregatício também evidenciaram diferença estatisticamente significativa entre as dimensões do JSS.

Diante disso, nota-se que a satisfação no trabalho é considerada um indicador de saúde do trabalhador, principalmente dos técnicos de enfermagem atuantes no atendimento pré-hospitalar. Isso posto, ressalta-se que a partir dos resultados deste trabalho, gestores e coordenadores de enfermagem não somente das unidades pesquisadas, como de outras unidades do SAMU, podem nortear suas ações de modo a promover/desenvolver os fatores de satisfação no trabalho com a equipe dos técnicos de enfermagem.

\section{REFERÊNCIAS}

1. Santos RR, Paiva MCMS, Spiri WC. Association between nurses' quality of life and work environment. Acta Paul. Enferm. [Internet]. 2018 [cited 2021 Mar 19]; 31 (5):472-9. DOI: https://doi.org/10.1590/1982-0194201800067.

2. Lu H, Zhao Y, While A. Job satisfaction among hospital nurses: A literature review. Int. J. Nurs. Stud. [Internet]. 2019 [cited 2021 Mar 19]. DOI: https://doi.org/10.1016/j.ijnurstu.2019.01.011.

3. Staempfli S, Lamarche K. Top ten: A model of dominating factors influencing job satisfaction of emergency nurses. Int. Emerg Nurs. 2020 [cited 2021 Mar 19] 1; 49:100814. DOI: https://doi.org/10.1016/j.ienj.2019.100814.

4. Liu Y, Aungsuroch Y, Yunibhand J. Job satisfaction in nursing: a concept analysis study. Int. Nurs. Rev. [Internet]. 2016 [cited 2019 Mar 19]; 63(1):84-91. DOI: https://doi.org/10.1111/inr.12215.

5. Souza AC de, Alexandre NMC, de Brito Guirardello E. Validation of the Brazilian Version of the Job Satisfaction Survey Using Confirmatory Factor Analysis. J. Nurs. Meas. [Internet]. 2017 [cited 2021 Mar 19]; 25(1):46E-65E. DOI: https://doi.org/10.1891/1061-3749.25.1.E46.

6. Locke EA. The nuture and causes of job satisfaction. In: Dunnette MD, editor. Handbook of industrial and organizational psychology. Rand McNal. Chicago; 1976. p. 1297-350.

7. Machado $\mathrm{MH}$, Filho WA, Lacerda WF, Oliveira $\mathrm{E}$, Lemos $\mathrm{W}$, Wermelinger $\mathrm{M}$, et al. Características gerais da enfermagem: o perfil sóciodemográfico. Enferm. em Foco [Internet]. 2016 [cited 2021 Mar 19]; 7(ESP):9-14. DOI: https://doi.org/10.21675/2357707X.2016.v7.nESP.686 
8. Wermelinger MCMW, Boanafina A, Machado MH, Vieira M, Ximenes Neto FRG, Lacerda WF. Nursing technician training: qualification profile. Cienc. e Saude Coletiva [Internet]. 2020 [cited 2021 Mar 19]; 25(1):67-78. DOI: http://dx.doi.org/10.1590/1413-81232020251.27652019.

9. Campos RM, Farias GM de, Ramos CDS. Satisfação profissional da equipe de enfermagem do SAMU/Natal. Rev. Eletrônica Enferm. [Internet]. 2009 [cited 2018 Feb 14]; 11(3):647-57. Available from: https://www.revistas.ufg.br/fen/article/view/47200/23140.

10. O'Dwyer G, Konder MT, Reciputti LP, Macedo C, Lopes MGM. Implementation of the Mobile Emergency Medical Service in Brazil: action strategies and structural dimension. Cad Saude Publica [Internet]. 2017 [cited 2021 Mar 20]; 33(7):e00043716. DOI: https://doi.org/10.1590/0102-311x00043716.

11. Melo EM, Assis EV, Feitosa ANA, Sousa MNA. Satisfação dos enfermeiros que trabalham na urgência e emergência. Rev. Interdiscip. em Saúde [Internet]. 2016 [cited 2021 Mar 20]; 3(1):54-70. Available from: http://interdisciplinaremsaude.com.br/Volume_9/Trabalho_04.pdf.

12. Alves M, Rocha TB, Ribeiro HCTC, Gomes GG, Brito MJM. Specificities of the nursing work in the mobile emergency care service of Belo Horizonte. Texto Context - Enferm. [Internet]. 2013 [cited 2021 Mar 20]; 22(1):208-15. DOI: https://doi.org/10.1590/S0104-07072013000100025.

13. Garcia AB, Dellaroza MSG, Haddad MCL, Pachemshy LR. Pleasure in nursing technicians working at an emergency unit of a public university hospital. Rev. Gaúcha Enferm. 2012; [cited Mar 20]; 33(2):153-9. DOI: https://doi.org/10.1590/S198314472012000200022.

14. Carvalho AEL, Frazão IS, Silva DMR, Andrade MS, Vasconcelos SC, Aquino JM. Stress of nursing professionals working in pre-hospital care. Rev. Bras. Enferm. [Internet]. 2020 [cited 2021 Mar 19];73(2). DOI: http://dx.doi.org/10.1590/0034-7167-2018-0660.

15. Spector PE. Measurement of human service staff satisfaction: development of the Job Satisfaction Survey. Am. J. Community Psychol. 1985 [cited 2021 Mar 19];13(6):693-713. DOI: http://dx.doi.org/10.1007/BF00929796

16. Souza AC de, Milani D, Alexandre NMC. Adaptação cultural de um instrumento para avaliar a satisfação no trabalho. Rev. Bras. Saúde Ocup. [Internet]. 2015 [cited 2021 May 20]; 40(132):219-27. DOI: http://dx.doi.org/10.1590/0303-7657000113715.

17. Mukaka MM. Statistics corner: A guide to appropriate use of correlation coefficient in medical research. Malawi Med. J. [Internet]. 2012 Sep [cited 2019 Feb 6];24(3):69-71. Available from: https://www.ncbi.nlm.nih.gov/pmc/articles/PMC3576830/pdf/MMJ2403-0069.pdf.

18. Sansoni J, De Caro W, Marucci AR, Sorrentino M, Mayner L, Lancia L. Nurses' Job satisfaction: an Italian study. Ann Ig. [Internet]. 2016 [cited 2021 Mar 20]; 28(1):58-69. DOI: https://doi.org/10.7416/ai.2016.2085.

19. Alharbi J, Wilson R, Woods C, Usher K. The factors influencing burnout and job satisfaction among critical care nurses: a study of Saudi critical care nurses. J. Nurs. Manag. [Internet]. 2016 [cited 2021 Mar 20]; 24(6):708-17. DOI: https://doi.org/10.1111/jonm.12386.

20. Skitsou A, Anastasiou M, Charalambous G, Andrioti D. Job Satisfaction of Nurses in a Psychiatric Hospital, in Cyprus. Int. J. Caring Sci. [Internet]. 2015 [cited 2021 Mar 20]; 8(3):683-97. Available from: http://www.internationaljournalofcaringsciences.org/docs/20_Skitsou_original_8_3.pdf.

21. Moura AA, Bernardes A, Balsanelli AP, Zanetti ACB, Gabriel CS. Leadership and nursing work satisfaction: An integrative review. ACTA Paul. Enferm. [Internet]. 2017; [cited 2021 Mar 20]; 30(4):442-50. DOI: http://dx.doi.org/10.1590/1982-0194201700055.

22. Freire C, Bettencourt C. Impact of ethical leadership on job satisfaction: the mediating effect of work-family conflict. Leadersh. Organ. Dev. J. [Internet]. 2020 [cited 2021 Mar 20]; 30:41(2):319-30. DOI: https://doi.org/10.1108/LODJ-07-2019-0338.

23. Aloisio LD, Baumbusch J, Estabrooks CA, Bostrom A, Chamberlain S, Cummings GG, et al. Factors affecting job satisfaction in long-term care unit managers, directors of care and facility administrators: A secondary analysis. J. Nurs. Manag. [Internet]. 2019 [cited 2021 Marr 20]; 27(8):1764-72. DOI: https://doi.org/10.1111/jonm.12871.

24. Moura AA, Bernardes A, Balsanelli AP, Dessotte CAM, Gabriel CS, Zanetti ACB. Leadership and job satisfaction in the Mobile Emergency Care Service context. Rev. Lat. Am. Enfermagem [Internet]. 2020 [cited 2021 Mar 20]; 28:e3260. DOI: https://doi.org/10.1590/1518-8345.3455.3260.

25. Foà C, Bertinelli S, Boschini A, Fragnelli M, Svichkar V, Tempone MR, et al. Care Case Managers' Job Satisfaction: a first contribution to the Italian validation of the Job Satisfaction Scale. Acta bio-medica [Internet]. 2016 [cited 2018 May 18]; 87(2S):38-48. Available from: http://www.mattioli1885journals.com/index.php/actabiomedica/article/view/5430.

26. Sarella Parra LH, Paravic Klijn T. Satisfaccion laboral en enfermeras/os que trabajan en el Sistema de Atencion Medica de Urgencia (SAMU). Cienc. y enfermería [Internet]. 2002 [cited 2021 Mar 20]; 8(2):37-48. DOI: http://dx.doi.org/10.4067/S071795532002000200005.

27. Chaves LDP, Mininel VA, Silva JAM da, Alves LR, Silva MF da, Camelo SHH. Supervisão de enfermagem para integralidade do cuidado. Rev. Bras. Enferm. [Internet]. 2017 [cited 2021 Mar 21]; 70(5): 1165-70. DOI: http://dx.doi.org/10.1590/0034-7167-2016-0491.

28. Halcomb E, Smyth E, McInnes S. Job satisfaction and career intentions of registered nurses in primary health care: an integrative review. BMC Fam. Pract. [Internet]. 2018 [cited Mar 20]; 19(1):1-14. DOI: https://doi.org/10.1186/s12875-018-0819-1.

29. Penconek T, Tate K, Bernardes A, Lee S, Micaroni SPM, Balsanelli AP, et al. Determinants of nurse manager job satisfaction: A systematic review. Int. J. Nurs. Stud. [Internet]. 2021 [cited 2021 Mar 22]; 118:103906. DOI: https://doi.org/10.1016/j.ijnurstu.2021.103906.

30. Wisniewski D, Silva ES, Évora YDM, Matsuda LM. Satisfação profissional da equipe de enfermagem $x$ condições e relações de trabalho: Estudo relacional. Texto e Context Enferm. [Internet]. 2015 [cited 2021 Mar 22]; 24(3):850-8. DOI: https://doi.org/10.1590/0104-070720150000110014. 